\title{
Initial Experience With Asynchronous Transfer Mode for Use in a Medical Imaging Network
}

\author{
Minh DoVan, Louis M. Humphrey, Geri Cox, and Carl E. Ravin
}

\begin{abstract}
Picture archiving and communication Systems (PACS) for medical imaging have always suffered from bandwidth limitations, throughput, and proprietary protocols. Commercially available local area networks have been hard pressed to meet the requirements of image transfer in a time consistent with patient-care needs. Recent technologic advances provide potential solutions to these constraints. Asynchronous transfer mode (ATM) provides the aggregate bandwidth and throughput that may be sufficient to satisfy the medical imaging community. Networks using prototype ATM technology have been available and commercial hardware is now becoming available. This report presents initial performance results of an ATM network and its suitability for use in a digital imaging network. Throughput of 10 Mbytes/sec was attained with Transmission Control Protocol/Internet Protocol using commercially available hardware.

Copyright 1995 by W.B. Saunders Company
\end{abstract}

KEY WORDS: network, picture archiving and communication system (PACS), image transmission, Transmission Control Protocol / Internet Protocol (TCP/IP), Digital Imaging and Communications in Medicine (DICOM).

$\mathbf{T}$ HE CONCEPT OF picture archiving and communication systems (PACS) has survived many setbacks. Lack of standards for acquisition and transport of images has always caused problems in retrieving images from digital imaging modalities such as computed tomography (CT) and magnetic resonance (MR). Storage requirements for radiologic image data, both short-term and long-term, are tremendous. Local area networks (LANs) and wide area networks (WANs) to support rapid transfers of image data have not been commercially available, but the need for this is apparent. Despite these obstacles, the compelling potential advantages of PACS have been so great as to sustain it.

The Digital Imaging and Communications in Medicine (DICOM) 3.0 protocol standard is beginning to aid in the acquisition and transport of images. In the past few years, large optical and tape archives have appeared as have architectures for distributed and centralized storage. Recently, products for a new networking technology, asynchronous transfer mode (ATM), were introduced for local and wide area communications. An ideal radiologic network must support high throughput transfer rates, carry video and voice, and extend seamlessly to a WAN. Although still in its early stages, ATM appears posed to fulfill these requirements.

Various high-speed networks have been built to support medical imaging needs, but most of these have been research oriented or of a proprietary nature. The current most popular network, Ethernet, does not provide sufficient bandwidth or throughput for clinical applications involving the transmission of medical images. Fiber distributed data interface (FDDI) is a marked improvement, providing much higher bandwidth, but like Ethernet, it is a shared media. In a shared-media environment, traffic from all computers on a network segment affects the total bandwidth utilization. Two computers transferring large amounts of data have the potential to saturate the entire network and thus deprive other computers of network bandwidth. In a model of a large clinical PACS, ATM appears to be better equipped to handle medical image-transmission needs. A major advantage of the circuit-based ATM network over the shared-media network is that traffic between two computers travels over a defined circuit as opposed to being transmitted over shared media (eg, Ethernet). Consequently, the aggregate bandwidth for the ATM network is much higher than just the point-to-point bandwidth. Two computers can saturate the ATM circuit between them, but this does not affect the bandwidth utilization of the entire network. Direct ATM connections used for high-performance imaging applications provide dedicated bandwidth for a workstation or server. This prevents imaging traffic from congesting an existing Ethernet network and also isolates imaging traffic from other ATM workstations.

From the Duke University Medical Center, Department of Radiology, Durham, NC 27710.

Address reprint requests to Minh DoVan, Department of Radiology, Duke University Medical Center, Erwin Rd, PO Box 3808, Durham, NC 27710

Copyright $\odot 1995$ by W.B. Saunders Company

0897-1889/95/0801-0006\$3.00/0 
In addition to its high bandwidth, wide area providers have begun marketing ATM connections. The Information Highway is one such wide area service. The emergence of ATM in the broadband area, most notably the Information Highway, will pave the future for ATM in telemedicine.

A total medical imaging network will not be constructed solely on ATM. It is vital that an ATM network maintain connectivity with existing Ethernet networks. We believe that there is a role for both Ethernet and ATM in medical imaging networks. Ethernet could be used mainly in the transmission of images from modalities to acquisition devices or storage devices, or in applications where performance is not critical. ATM can be used most effectively for transmission of images from storage to display or wherever network performance is critical. ATM and Ethernet should operate as one homogenous network to provide transparent access to all imaging devices.

In this paper, we measure the performance of an ATM LAN and attempt to assess its viability for use in a radiologic imaging network. We have limited our tests to Transmission Control Protocol/Internet Protocol (TCP/IP) traffic because of its support in the DICOM 3.0 specification. As DICOM becomes widely accepted in the radiology community, performance of TCP/IP over ATM will be of great interest. Even though TCP/IP is not the most efficient protocol for ATM, it will probably be the most widely used. Our tests of TCP/IP over ATM yielded very satisfactory results with a few small surprises.

\section{MATERIALS AND METHODS}

Our test suite consisted of three ATM switches* and three Sparc 10 51s (single processor). One Sparc 10 (Sun S10GX-51; Sun Microsystems Inc, Mountain View, CA), via connection to the Ethernet port on one of the switches, downloads configuration information to the switches and controls the signaling in the ATM network. The other two Sparc 10 computers (Sun S10SX-51) have direct ATM connections to the switches using the user network interface (UNI) specification. $\dagger^{1}$ All the ATM interfaces operate at the $\mathrm{OC} 3 \mathrm{C}$ rate of $155 \mathrm{Mbps}$. One of the latter Sparcstations

*The ATM switches have 16 multimode SONET fiber ports (155 Mbps) (model 10114 from Synoptics).

†The Sparcstations are equipped with Synoptics SBUS ATM host interface (SAHI) adapters. also has an Ethernet adapter and operates as a router to provide connectivity between our ATM and Ethernet networks. This will be replaced in the future by an Ethernet switch that has Ethernet and ATM connections (EtherCell; Synoptics Communications Inc, Santa Clara, CA). Figure 1 depicts this configuration. The thin lines represent the Ethernet network and the thick lines represent the ATM fiber connections.

We performed a number of transfer tests using various utilities. Our purpose was to determine the throughput rates achievable using standard off-the-shelf hardware. This is a prime concern in the context of a medical imaging network. The results are actual transfer measurements and not simulations. These tests were performed with a focus on a medical imaging application where large volumes of data are transferred. As such, the results presented should indicate the performance to be expected in a clinical imaging application.

When performing the transfer tests, we used switched virtual circuits (SVCs) as opposed to permanent virtual circuits (PVCs). These are the two current methods of establishing an ATM connection. PVCs are permanent and must be manually constructed and removed. The standards on PVCs have been adopted and should provide current interoperability between different ATM manufacturers (provided the rates are the same). SVCs are established on a need basis (much like making a phone call). The standards on SVCs have not been set. As such, interoperability with SVCs probably will not happen until late 1994 (or 1995). Our reasons for using SVCs as opposed to PVCs were the simplicity with which we were able to establish SVC connections and the expectation that SVCs would be the typical method of connection for most PACS applications.

TCP/IP was tested using the standard file transfer protocol (FTP) and remote copy (RCP) utilities as well as the publicly available test tool ttcp and a special tool we developed to test transfers of medical images over an ATM network, atmtest (similar to ttcp). For each test, 10 transfers were made and the minimum, maximum, and average throughput measurements from the transmitting workstation were recorded. Each test was executed with normal priority (the UNIX nice command was not used). Graphs of the results are also presented.

FTP and RCP represent very popular file transfer utilities in a TCP/IP environment. They are useful in an imaging network for ad hoc transfers of images. A clinical PACS would not use FTP and RCP to transfer images, but rather the DICOM protocol. Without considering the DICOM

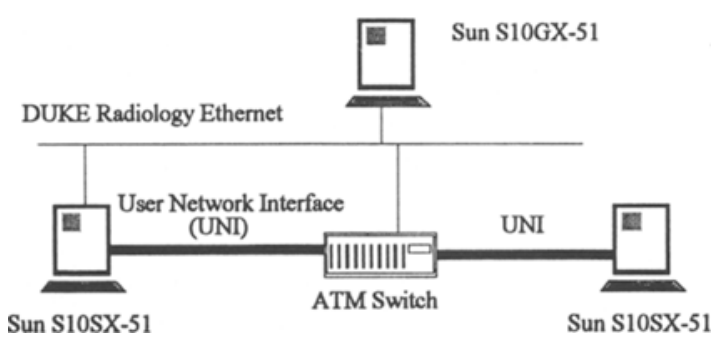

Fig 1. TCP/IP over ATM test configuration using one ATM switch. 
protocol overhead, the ttcp and atmtest tools provide a better view of the performance to be expected from a PACS using an ATM network.

The atmtest and ttcp test programs simply copy a static buffer across the network. The system time is recorded before the actual transfer and once again after the transfer is complete. Throughput rate is attained by dividing the amount of data transferred by the difference in the two times (which is performed by the test programs). Our atmtest program also has the ability to transfer large image files (where packets are read into sequential memory locations). In the ttcp test tool, a buffer is copied into the same memory location continuously. While this is a good measurement of performance, it does not stimulate an actual image transfer. Performance of FTP was recorded from the statistics produced after the file transfer is complete. RCP performance was manually calculated by dividing the amount of data transferred by the lapse in time recorded by the UNIX time command. Ten-megabyte files were used when testing file transfer (this size represents a typical 2,000- $\times 2,000-\times 12$-bit digital chest radiograph).

All tests were run with the two Sparcstations connected to one ATM switch. This would yield best-case results for ATM in LANs. The atmtest portion was also repeated with an additional ATM switch. One Sparcstation was connected to one switch (UNI), the second Sparcstation was connected to the other switch, and the two switches were connected (network node interface) as shown in Fig 2.

\section{RESULTS}

As discussed previously, ${ }^{2}$ the performance of TCP/IP depends upon the product of the transfer rate and the round-trip delay. As this product increases, the buffer space required must also increase. This became readily apparent during our initial tests where TCP/IP performance was in the 250 to $500 \mathrm{Kbyte} / \mathrm{s}$ ( 2 to 4 Mbps) range.

For high-performance throughput, it is necessary to properly tune the buffer sizes at the end

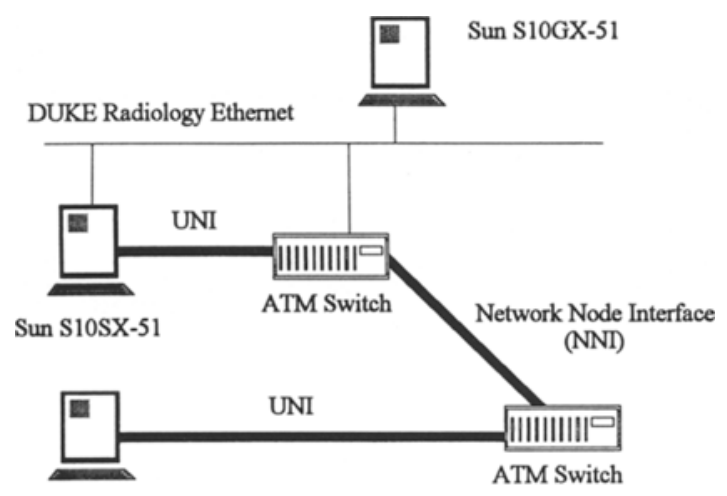

Sun S10SX-51

Fig 2. TCP/IP over ATM test configuration using two ATM switches.

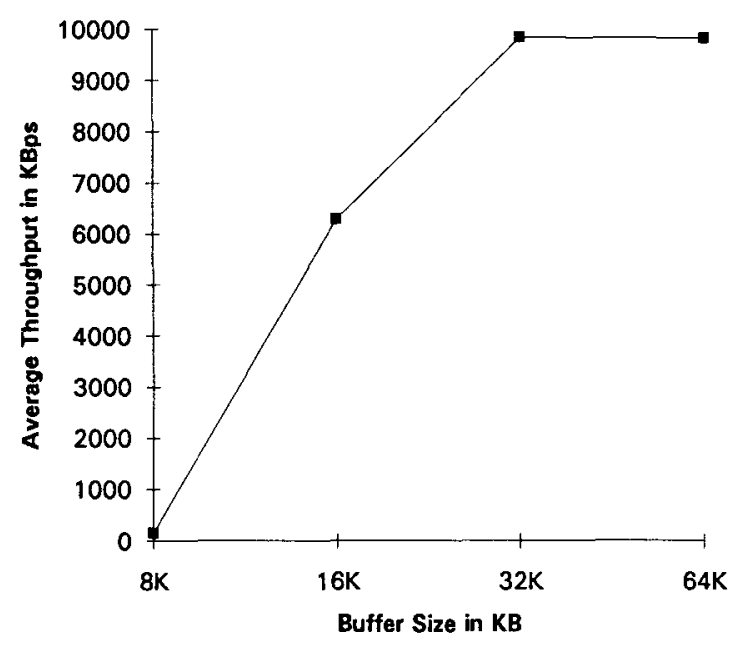

Fig 3. Throughput of TCP/IP over ATM versus socket buffer size.

workstations. A graph of buffer size versus throughput is shown in Fig 3. Our highest throughput measurements were in the 10 Mbyte/s ( $80 \mathrm{Mbps}$ ) range. Both the atmtest and ttcp test tools produced similar results.

As can be seen in Fig 4 and 5, there is a reduction in throughput when transferring actual image files. Figure 4 indicates the performance results when buffers are read into the same memory location and Fig 5 indicates the performance when buffers are read into sequential memory locations. We noticed no difference in the transfer rates of intraswitch and interswitch traffic as shown in Fig 6.

FTP and RCP performance were well below expectations as shown in Figs 7 through 10. A probable cause for this was a buffering problem in the operating system (or application program). A buffer that is too small will fill up and cause an artificial degradation in performance. To work around this, we repeated the tests using a random access memory (RAM) disk that should provide best-case results for FTP and RCP (/tmp may be used on Solaris [Sun Microsystems] with tmpfs). The speed of the RAM disk should prevent even small buffers from filling. A summary of these tests is shown in Table 1.

\section{DISCUSSION}

Our initial tests transferring images using TCP/IP over ATM (at $155 \mathrm{Mbps)}$ were very promising. Interestingly enough, the limitation 


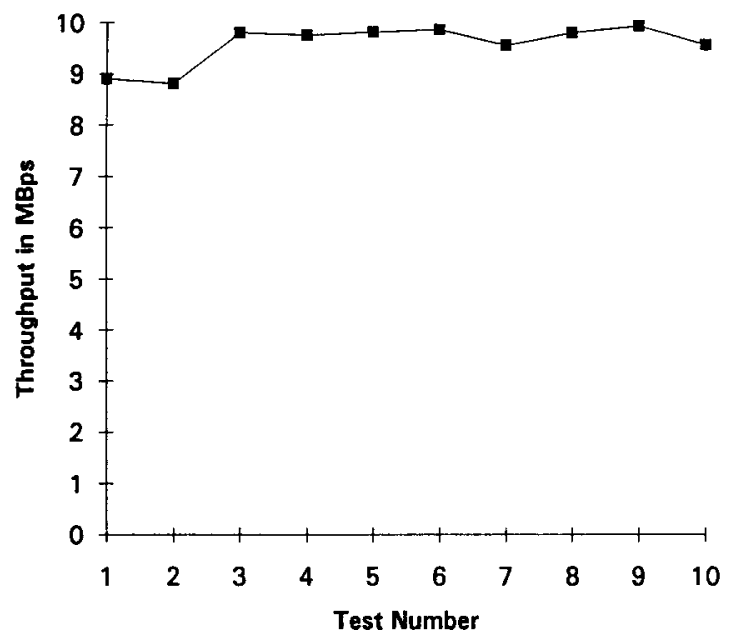

Fig 4. Variations in throughput of raw data transfer using TCP / IP over ATM.

on throughput was not the ATM network. The same tests run on only one machine (ie, loopback tests) produced similar results (actually, the loop-back performance was worse, probably because of two processes running on the same machine). At the time the tests were run, both performance meters showed central processing unit (CPU) utilization at $100 \%$ (when the transfer rates were $10 \mathrm{Mbyte} / \mathrm{s}$ ). This data implies that the limiting factor on throughput was the two end workstations and not the ATM link. In particular, the bottleneck appeared to be the CPU and processing of the TCP/IP protocol.

The ATM switches were able to transmit data

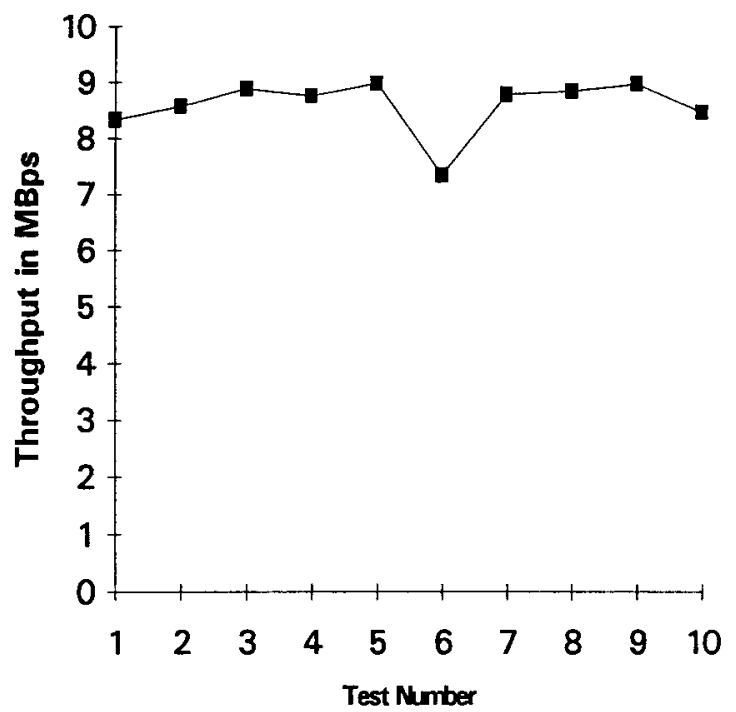

Fig 5. Variations in throughput of image data transfer using TCP/IP over ATM and 10-Mbyte image files.

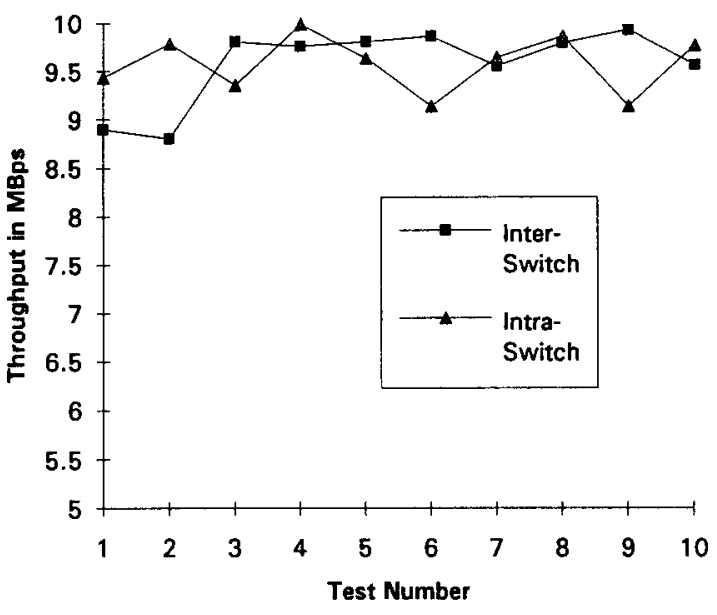

Fig 6. Comparison of intraswitch versus interswitch throughput using TCP/IP over ATM.

as fast as the computers could place data onto the network. We do not conclude that this is the limiting factor on throughput of these workstations. With some more work on enhanced drivers (ie, multithreaded), proper kernel tuning, and TCP/IP stack optimizations, these numbers should increase. Faster processors or dual processors should also increase performance.

We were able to attain rates of $10 \mathrm{Mbyte} / \mathrm{s}$ using standard hardware and unmodified operating systems with our test programs which transmitted data from one machine to another. We expect that these throughput measurements will not change drastically when the ATM network is used in a clinical setting as opposed to this experimental test bed. There will be some slight degradation caused by the overhead

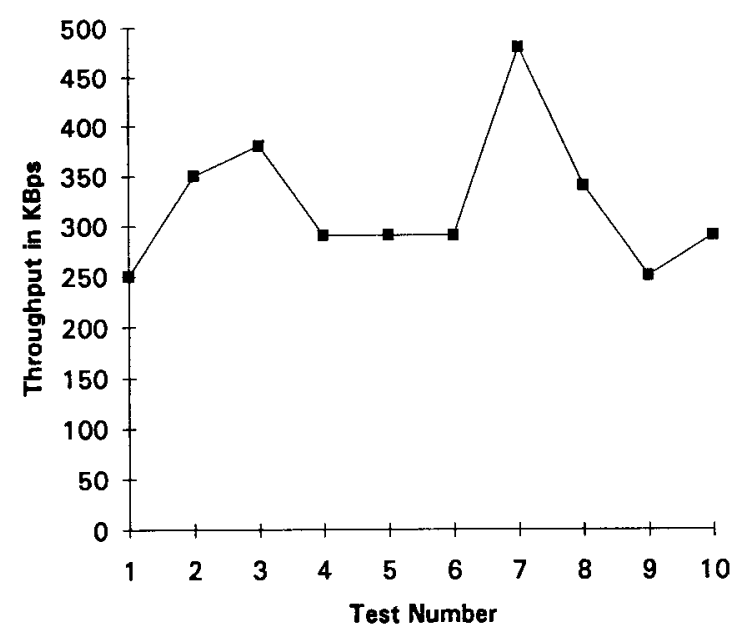

Fig 7. FTP throughput of disk-to-disk copies over ATM using 10-Mbyte image files. 


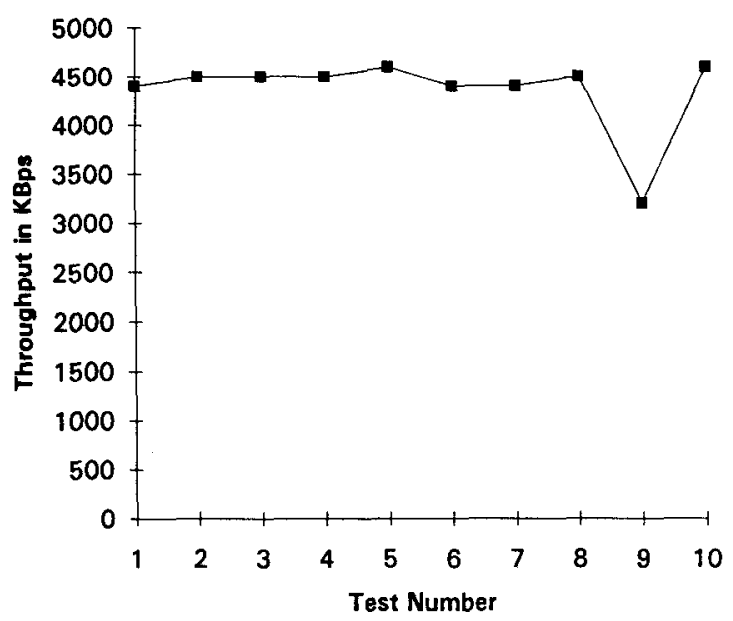

Fig 8. FTP throughput of RAM-disk-to-RAM-disk copies over ATM using 10-Mbyte image files.

of the DICOM 3.0 protocol. A realistic expectation would be throughput in the 7 to $8 \mathrm{Mbyte} / \mathrm{s}$ range. This will be dependent on the CPU utilization of the workstations. Several workstations requesting images from a central server may impact performance to a workstation because of the extra load and available bandwidth on the server. However, overall network performance should not be affected because of the circuit-based connections in the ATM environment.

FTP and RCP performance were surprising at an average of $\sim 300 \mathrm{Kbyte} / \mathrm{s}(2.4 \mathrm{Mbps})$. However, the tests of FTP and RCP from a RAM disk to a RAM disk indicated performance averaged above $4 \mathrm{Mbyte} / \mathrm{s}$ ( $32 \mathrm{Mbps}$ ) for FTP and slightly under 3 Mbyte/s (24 Mbps) for

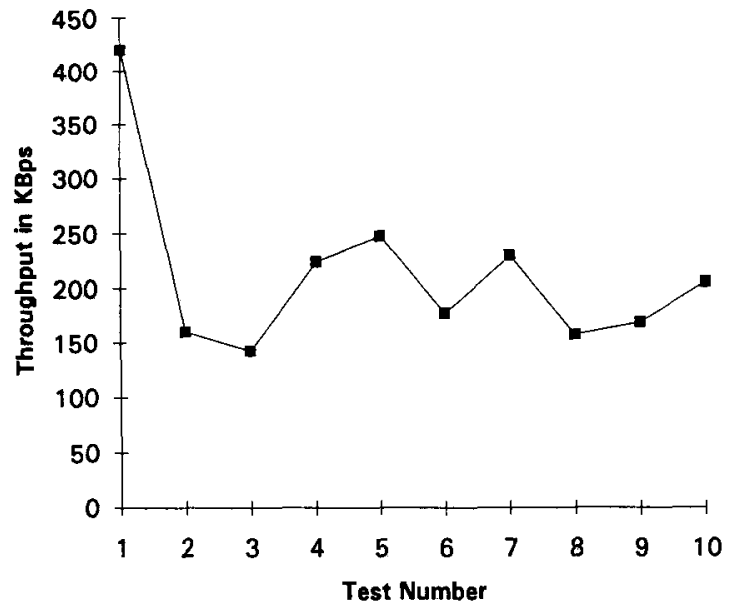

Fig 9. RCP throughput of disk-to-disk copies over ATM using 10-Mbyte image files.

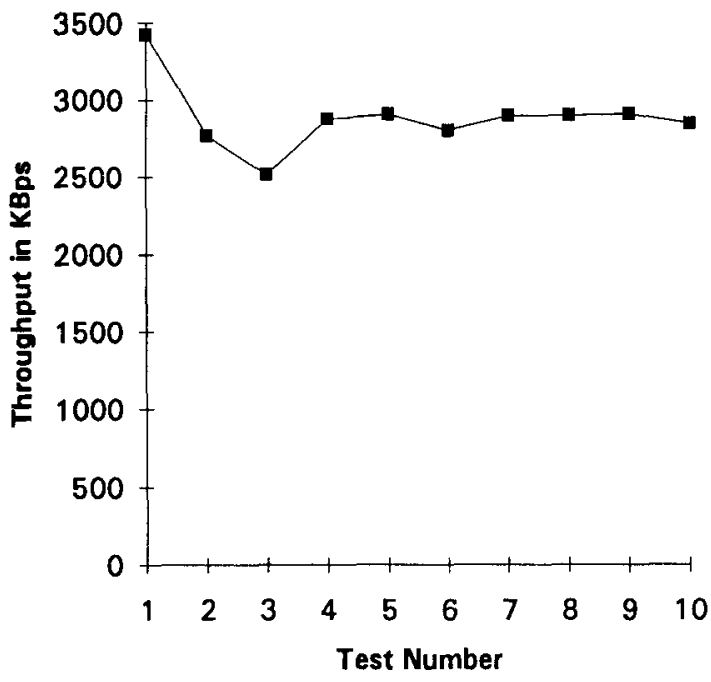

Fig 10. RCP throughput of RAM-disk-to-RAM-disk copies over ATM using 10-Mbyte files.

RCP. Clearly, proper tuning (or possibly operating system patches) will be necessary to attain high throughput rates.

Connectivity between Ethernet and ATM will be of vital importance in a medical imaging network. Currently, there are many imaging computers that support Ethernet from modalities to display stations. The ability to transfer image data between Ethernet and ATM will be imperative. We were able to tie our Ethernet and ATM networks together using one of the Sun workstations. In the future, this will be accomplished by Ethernet-to-ATM products. Manufacturers are also beginning to support segmentation and virtual LANs over ATM. With this, a network administrator can logically partition an Ethernet network without physical constraints because segments can span across the ATM switches. This is important in a large imaging network where several modalities can cause congestion on the Ethernet. One possible use of segmentation would be to place CT, MR,

Table 1. Summary of Performance Data

\begin{tabular}{lccc}
\hline & $\begin{array}{c}\text { Minimum } \\
\text { (Mbytes/s) }\end{array}$ & $\begin{array}{c}\text { Maximum } \\
\text { (Mbyte/s) }\end{array}$ & $\begin{array}{c}\text { Average } \\
\text { (Mbyte/s) }\end{array}$ \\
\hline Raw transfer & 8.81 & 9.93 & 9.58 \\
Image transfer & 7.33 & 8.98 & 8.59 \\
Interswitch transfers & 9.13 & 9.99 & 9.58 \\
FTP (Disk I/O) & 0.25 & 0.48 & 0.32 \\
FTP (RAM disk I/O) & 3.20 & 4.60 & 4.36 \\
RCP (Disk I/O) & 0.14 & 0.42 & 0.21 \\
RCP (RAM disk I/O) & 2.51 & 3.42 & 2.88
\end{tabular}


ultrasound, and computed radiography machines on individual segments.

Congestion is always a concern in networks. However, the current model of congestion of TCP/IP will change in a circuit-based ATM environment. Because IP traffic does not affect other circuits, IP congestion will be limited to the number of concurrent sessions on a workstation (as opposed to all traffic in a shared media environment). This provides ATM a strong advantage in a medical imaging network and is important when considering aggregate bandwidth. Because FDDI (Token-Ring), Ethernet, and 100-Mbps Ethernet (Carrier Sense Multiple Access with Collision Detection) are shared media, they do not offer the virtual throughput and scalability of ATM. For example, consider multiple imaging workstations transmitting large image studies at $80 \mathrm{Mbps}$ (as in our current tests). This will become commonplace in the near future. Both FDDI and 100-Mbps Ethernet will become congested. ATM, because of its circuit-based connections, should handle multiple, concurrent transmissions at this rate with ease.

Many of the wide area providers have strategies using ATM technology. This makes ATM a logical choice when remote high-bandwidth connections are planned. Connections to the Information Highway and telemedicine applications (using voice, video, and data) are currently planned. To be successful in the wide area will require interoperability. ATM is still in its infancy and interoperability is always of concern during the early stages, but this work is advancing at a rapid rate. It is important to understand these interoperability issues when pursuing ATM today.

These tests of commercially available ATM hardware indicate that high throughput is achievable today using TCP/IP. Proper tuning of the end workstations will be a major factor in achieving this throughput. This high throughput alone would make ATM a good choice for an imaging network. In addition, because of the circuit-based connections, the aggregate bandwidth of the network is much higher than just the point-to-point bandwidth. The high throughput rates, switch-based architecture, capacity to carry video and voice, and the evolution of ATM into the wide area makes ATM a viable choice for a medical imaging network.

\section{REFERENCES}

1. The ATM Forum: ATM User-Network Interface Specification. Englewood Cliffs, NJ, Prentice-Hall, 1993, pp 2-10
2. Jacobson V, Braden, R, Borman D: TCP extensions for high performance, RFC 1323. LBL, ISI, and Cray Research, May 1992, pp 2-3 